\title{
Role of mono-ADP-ribosylation histone modification (Review)
}

\author{
JING-JING ZHA $^{1^{*}}$, YI TANG $^{2 *}$ and YA-LAN WANG ${ }^{2}$ \\ ${ }^{1}$ Pathological Department, First Affiliated Hospital of Chongqing Medical University; ${ }^{2}$ Department of Pathology, \\ Molecular Medicine and Cancer Research Center, Chongqing Medical University, Chongqing 400016, P.R. China
}

Received August 26, 2020; Accepted February 22, 2021

DOI: $10.3892 /$ etm.2021.10009

\begin{abstract}
The current knowledge regarding ADP-ribosylation modifications of histones, particularly mono-ADP-ribosylation modifications, is limited.However, recent studies have identified an increasing number of mono-ADP-ribosyltransferases and the role of mono-ADP-ribosylation has become a hot research topic. In particular, histones that are substrates of several mono-ADP-ribosyltransferases and mono-ADP-ribosylated histones were indicated tobe involved in numerous physiological or pathological processes. Compared to poly-ADP-ribosylation histone modification, the use of mono-ADP-ribosylation histone modification is restricted by the limited methods for research into its function in physiological or pathological processes. The aim of the present review was to discuss the details regarding mono-ADP-ribosylation modification of histones and the currently known functions thereof, such as cell physiological and pathological processes, including tumorigenesis.
\end{abstract}

\section{Contents}

1. Introduction

2. Enzymes of mono-ADP-ribosylation in mammals

3. Histones are substrates of mono-ADP-ribosylation

4. Methods for detecting mono-ADP-ribosylated histones

5. Function of mono-ADP-ribosylated histones in DNA damage and repair

6. Mono-ADP-ribosylation of histones in gene replication and transcription

7. Mono-ADP-ribosylation of histones in cell proliferation and differentiantion

Correspondence to: Professor Ya-Lan Wang, Department of Pathology, Molecular Medicine and Cancer Research Center, Chongqing Medical University, 1 Medical College Road, Yuzhong, Chongqing 400016, P.R. China

E-mail: wangyalan@cqmu.edu.cn

${ }^{*}$ Contributed equally

Key words: mono-ADP-ribosylation, histone, epigenetics
8. Association between mono-ADP-ribosylation of histones and other histone modifications

9. Hydrolytic enzymes of histone mono-ADP-ribosylation

10. Conclusion

\section{Introduction}

The occurrence and development of cancer may involve genetic as well as epigenetic changes (1). The complex processes of carcinogenesis cannot be fully explained by genetic mutations alone, as they also involve epigenetic alterations. Epigenetics, including DNA methylation, RNA editing, genomic imprinting and post-translational histone modifications, is a branch of genetics that investigates changes in gene expression without alterations in the primary DNA sequence. Abnormal epigenetic processes regulate gene expression, alter gene function and promote tumorigenesis. Epigenetics is also widely reported to have an important role in the early stages of neoplastic development and cancer progression (2). While the early focus was on the DNA sequence as a critical epigenetic marker in the progression of cancer, an increasing number of subsequent studies have been focusing on the function of histone modifications in tumorigenesis $(3,4)$. Histone modifications include acetylation, phosphorylation, methylation, ADP-ribosylation, ubiquitylation and sumoylation, among which acetylation of histones has already been confirmed to be involved in the regulation of various types of cancer. As more mono-ADP-ribosyltransferases have been identified in recent years, the functions of mono-ADP-ribosylation of histones in human disease development, including cancer, have been further elucidated.

Mono-ADP-ribosyltransferase has been indicated to transfer one ADP-ribose from the co-factor $\mathrm{NAD}^{+}$to target proteins (Fig. 1), and it has been hypothesized that there are $\sim 1,000$ mono-ADP-ribosylated proteins in cells (5). Several mono-ADP-ribosylated proteins, such as NF- $\mathrm{BB}$ essential modulator (6), inositol-requiring enzyme $1 \alpha$, proline extensin-like receptor kinase1 (7), histones, RhoA and human $\alpha$-defensin 1 (8) have been identified to be involved in regulating immunity, inflammation and the stress response. However, there is still a lack of understanding of the functions of most of these mono-ADP-ribosylated proteins due to limitations in the methods or the tools used.

Histones, a type of basic proteins that combine with DNA in the chromosome, include five types in eukaryotes, namely 
histone (H)1, H2A, H2B, H3 and $\mathrm{H} 4$ (4 types of core histones). A number of studies have indicated that poly-ADP-ribosylation of histones have important roles in DNA repair, replication, transcription $(9,10)$, cell proliferation (11) and cancer, and may be associated with histone acetylation (12), methylation (13) and phosphorylation (14). However, there is a lack of research into the function of mono-ADP-ribosylation in physiological or pathological processes. The number of mono-ADP-ribosyltransferase types that have been detected in mammals has increased and these enzymes have been indicated to catalyze mono-ADP-ribosylation of histones. Mono-ADP-ribosylation of histones has also been associated with other modifications. The functions of mono-ADP-ribosylation of histones may comprise roles in important physiological pathways, which may include the development of malignant tumors (15).

\section{Enzymes of mono-ADP-ribosylation in mammals}

Approximately 22 members of the ADP-ribosyltransferase (ART) superfamily have been identified and have been indicated to have diverse roles. Certain ARTs modify proteins with chains of poly ADP-ribose or with mono ADP-ribose (mADPr) (Table I). According to the different structures of the catalytic domains, ARTs are divided into bacterial diphtheria toxin-like ARTs (ARTDs) (16) and clostridial C2 and C3 toxin-like ARTs (ARTCs) (17). ARTDs, which were previously termed the poly(ADP-ribose) polymerase (PARP) family and include 17 members $(18,19)$, are widely distributed in cells and are mostly concentrated in the nucleus. Compared with ARTD1-6 possessing, ARTD7-17 (except ARTD13, which is catalytically inactive) only catalyzes mono-ADP-ribosylation $(16,20)$, due to the absence of conserved glutamate. In addition, ARTD3 has been detected as a mono-ADP-ribosyltransferase in previous studies (21). ARTCs, as ectocellular ARTs, transfer mADPr from NAD ${ }^{+}$ to target proteins in the cytoplasm, cytomembrane and extracellular regions $(22,23)$. Therefore, ARTCs are not able to mediate mono-ADP-ribosylation of histones, which are distributed in the nucleus. Apart from the aforementioned, members of the sirtuin (SIRT) family (SIRT1-7 always act as histone deacetylases) possess mono-ADP-ribosylation properties. SIRT4, as well as SIRT6 and -7, were indicated to have endogenous mono-ADP-ribosyltransferase activity in the mitochondria and the nucleus, respectively (24-27). However, only a small number of these mono-ADP-ribosyltransferases, such as ARTD3, -10, and -14 and SIRT4 and -6 , have been reported to mono-ADP-ribosylate histones in vertebrates to date (28-32).

\section{Histones are substrates of mono-ADP-ribosylation}

All core histones and the linker histone $\mathrm{H} 1$ have been reported to undergo mono-ADP-ribosylated modification (Table II) (33). Histone $\mathrm{H} 1$ was indicated to be mono-ADP-ribosylated on glutamic acid E2, E14 and E116 (34), the arginine residue $\mathrm{R} 34$ (35) and at the $\mathrm{COOH}$-terminal lysine residue $\mathrm{K} 213$ (36). In the rat liver, histone H2B was indicated to be mono-ADP-ribosylated on glutamate residue 2 of the $\gamma-\mathrm{COOH}$ group (37). Both in the chromatin and in the reconstituted recombinant nucleosomes of chicken, histone H2B E2 was a specific target of PARP3 modification with mADPr (30). In histone $\mathrm{H} 2 \mathrm{~B}$, the residues E18 and E19 were also indicated to be the principal sites modified by the ARTs in response to DNA double-strand breaks (38). After hepatoma cells are alkylated, $\mathrm{H} 1$ and $\mathrm{H} 2 \mathrm{~B}$ may be mono-ADP-ribosylated at the N-terminal fragment. Mono-ADP-ribosylation also modified the Arg residues of $\mathrm{H} 2 \mathrm{~A}, \mathrm{H} 3$ and $\mathrm{H} 4$ and the glutamic residues of $\mathrm{H} 2 \mathrm{~A}$ and H2B (39). Previous studies identified K13 of H2A, K30 of $\mathrm{H} 2 \mathrm{~B}, \mathrm{~K} 27$ and $\mathrm{K} 37$ of $\mathrm{H} 3$ and $\mathrm{K} 16$ of $\mathrm{H} 4$ as ADP-ribose acceptor sites in poly-ADP-ribosylation (40); however, whether these are also ADP-ribose acceptor sites in mono-ADP-ribosylation requires further research. Therefore, the specific amino acid sites of mono-ADP-ribosylated modifications on H2A and $\mathrm{H} 4$ have remained elusive. It has remained to be determined which enzymes mediate mono-ADP-ribosylation of histones. With ongoing research, an increasing number of studies have identified specific enzymes involved in regulating the mono-ADP-ribosylation of histones. Human H2B was reported to be modified on E2 in vitro by using recombinant ARTD10 (41) and DNA damage was able to induce mono-ADP-ribosylation of H2B E18 and E19 in vivo by specific ARTs: Protein (ADP-ribosyl) transferase (ADPRT)1a and ADPRT2 (38). In addition, a silent information regulator 2 of yeast (SIR2)-related protein from the protozoan parasite Trypanosoma brucei (TBSIR2RP1), was indicated to catalyze mono-ADP-ribosylation of $\mathrm{H} 2 \mathrm{~A}, \mathrm{H} 2 \mathrm{~B}$ and H4 in Trypanosoma brucei (42).

\section{Methods for detecting mono-ADP-ribosylated histones}

The detection of the enzymes and specific substrates of ADP ribosylation is an important step for identifying mono-ADP-ribosylated histones. Radiolabeled or chemically modified $\mathrm{NAD}^{+}$has been widely used for detecting mono-ADP-ribosylated proteins in vitro. In the process of ADP ribosylation, radioactive-labeled $\mathrm{ADP}$ ribose of $\mathrm{NAD}^{+}$combines with the target proteins and SDS-PAGE autoradiography may detect these radiolabeled molecules (43). Certain studies have used self-made ADP-ribosylated antibodies, as commercially available specific antibodies are limited; however, the sensitivity of the antibodies has not been satisfactory and they are not generally applicable for wider use $(44,45)$. Osago et al $(46)$ have already investigated more efficient antibodies, which were able to even detect specific arginine mono-ADP-ribosylated peptides. Apart from the methods mentioned above, certain other chemical tools may be used to detect ADP-ribosylated proteins, even mono-ADP-ribosylated histones, such as the use of ADPr (ADP-ribose)-peptides, analogues (ADPr-synthon) and ADPr-chains (47). There are several chemical synthesis peptides carrying mADPr, which may be used to detect the affinity between ADP-ribose and substrates (48). In addition, the incorporation of benzophenone photo-cross-linkers into synthetic peptides has been demonstrated to provide a way to probe for and enrich ADP-ribose binding proteins $(49,50)$. As the methods for ADP-ribose peptide synthesis have improved, the development of corresponding antibodies may be possible (51). Thus, the specific antibody for histone mono-ADP-ribosylation may be improved to further investigate the function of histones in different fields.

Mass spectrometry (MS) and selective reader domains have also been used for detecting mono-ADP-ribosylated 


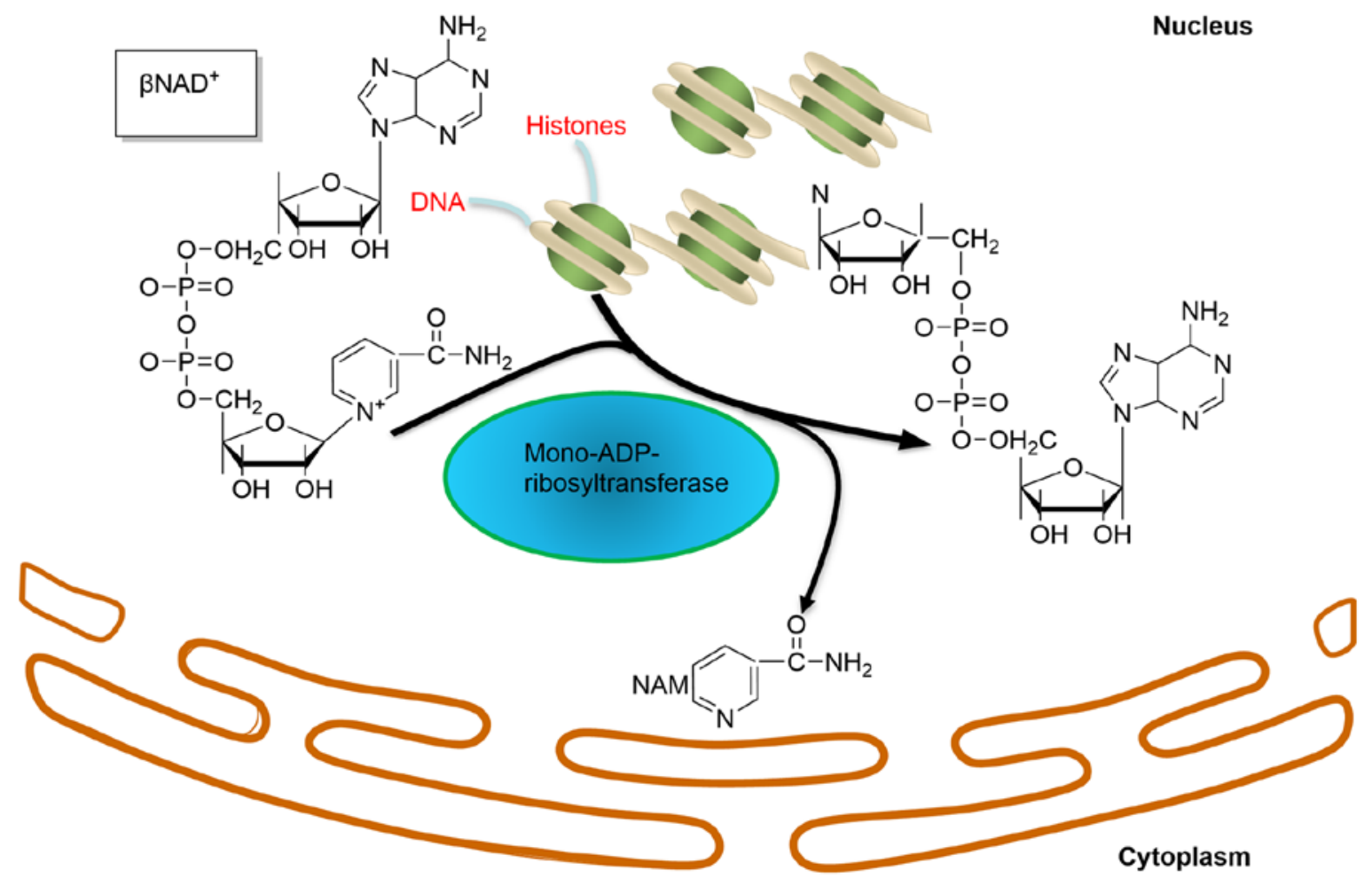

Figure 1. Schematic representation of the histone mono-ADP-ribosylation reaction.

proteins by identifying macrodomains, which may selectively bind ADP-ribose. H2A, one of a number of types of macrodomain-containing proteins, contains macro $\mathrm{H} 2 \mathrm{~A}$, which is subdivided into macro H2A1.1, macro H2A1.2 and macro H2A2 (52,53). Only H2A1.1 has been indicated not to bind mono-ADP-ribose (54), while it has remained to be determined whether mono-ADP-ribose has connections with macro H2A1.2 and macro H2A2 (52). Therefore, MS may be an important method for identifying mono-ADP-ribosylated histones. For detecting the specific ADP-ribosylated residues, quadruple tandem $\mathrm{MS}$ was indicated to detect ADP-ribosyl-Arg and Arg-ADP-ribosylated peptides to identify the specific arginine site of mono-ADP-ribosylation in the target protein $(55,56)$. In recent years, numerous MS-based proteomics have been developed, such as macrodomain-linked immunosorbent assay to identify mono-ARTs (57) and liquid chromatography-high-resolution tandem MS to identify mono-ADP-ribose acceptor sites $(58,59)$. Furthermore, there are other methods allowing for the identification of mono-ADP-ribosylation, such as a phosphoproteomics approach via the enzymatic product of phosphodiesterase-treated ADP-ribose (60) or an aminooxy alkyne probe for detecting mono-ADP-ribosylated proteins (61). A mutagenesis approach has also been employed to detect the ADP-ribose acceptor site (5), which may be a novel way to study mono-ADP-ribosylation of histones.

\section{Function of mono-ADP-ribosylated histones in DNA damage and repair}

Mono-ADP-ribosylation of histones may be involved in DNA damage and repair (62) and the nucleosomal surface is the main target (63). In the 1980s, Adamietz and Rudolph (64) reported that, when AH7974 hepatoma cells were damaged by the alkylating agent dimethyl sulfate, mono-ADP-ribosylation of histones increased by a factor of 12 . Under the same conditions, the mono-ADP-ribosylated C-terminal extension of histone $\mathrm{H} 1$ and the N-terminal fragment of histone $\mathrm{H} 2 \mathrm{~B}$ was increased compared with that in untreated cells $(65,66)$, which may modify DNA-histone association by adding two negative charges. TbSIR2RP1, catalyze the mono-ADP-modification of $\mathrm{H} 2 \mathrm{~A}$ and $\mathrm{H} 2 \mathrm{~B}$, which may occur in response to DNA damage and be involved in DNA repair. Rulten et al (67) suggested that mono-ADP-ribosylated H1, catalyzed by PARP3, may accelerate DNA double-strand break repair by binding to aprataxin and polynucleotide kinase-like factor. Changes in the types of ADP-ribosylated histones may occur in DNA strand breaks, as in the P815 mouse mastocytoma and K562 human chronic myelogenous leukemia cell lines, mono-ADP-ribosylated histones appeared in the absence of DNA strand breaks due to the decrease of poly-ADP-ribose synthetase activity, whereas poly-ADP-ribosylated histones increased following DNA stand breaks (68).

\section{Mono-ADP-ribosylation of histones in replication and transcription}

ADP ribosylation of histone is also involved in DNA repair and replication. It has been indicated that histones are predominantly mono-ADP-ribosylated in lysates of non-dividing cells, while being poly-ADP-ribosylated in rapidly proliferating cells $(62,69)$. However, evidence for the connection between mono-ADP-ribosylation of histones and replication is limited. Mono-ADP-ribosylated histones present in the nuclei under 
Table I. Enzymes of ADP-ribosylation.

\begin{tabular}{lccl}
\hline A, ARTDs & & & \\
\hline Enzymes & Subcellular localization & Enzymatic activities & $($ Poly-ADP-ribosyltransferase \\
\hline ARTD1 & Nucleus & Poly-ADP-ribosyltransferase & $(16-19)$ \\
ARTD2 & Nucleus & Mono/Poly-ADP-ribosyltransferase \\
ARTD3 & Nucleus & Poly-ADP-ribosyltransferase & $(16-19)$ \\
ARTD4 & Nucleus/cytoplasm & Poly-ADP-ribosyltransferase & $(22,31)$ \\
ARTD5 & Nucleus/cytoplasm & Poly-ADP-ribosyltransferase & $(16-19)$ \\
ARTD6 & Nucleus/cytoplasm & Mono/Poly-ADP-ribosyltransferase \\
ARTD7 & Nucleus & Mono/Poly-ADP-ribosyltransferase & $(16-19)$ \\
ARTD8 & Nucleus/cytoplasm & Mono/Poly-ADP-ribosyltransferase & $(16-19)$ \\
ARTD9 & Nucleus/cytoplasm & Mono/Poly-ADP-ribosyltransferase & $(20,21)$ \\
ARTD10 & Nucleus/cytoplasm & Mono/Poly-ADP-ribosyltransferase & $(20,21)$ \\
ARTD11 & - & Mono/Poly-ADP-ribosyltransferase & $(19)$ \\
ARTD12 & Nucleus & Catalytically inactive & $(20,21,30,42)$ \\
ARTD13 & Nucleus & Mono/Poly-ADP-ribosyltransferase & $(19-21)$ \\
ARTD14 & Nucleus & Mono/Poly-ADP-ribosyltransferase & $(19,29)$ \\
ARTD15 & Nucleus/cytoplasm & Mono/Poly-ADP-ribosyltransferase & $(20,21)$ \\
ARTD16 & - & Mono/Poly-ADP-ribosyltransferase & $(20,21)$ \\
ARTD17 & - & & $(20,21)$ \\
\hline
\end{tabular}

B, ARTCs

\begin{tabular}{lcc}
\hline Enzymes & Subcellular localization & Enzymatic activities \\
\hline ARTC1-5 & Ecto-cellular & - \\
\hline
\end{tabular}

C, special ARTs

\begin{tabular}{lcll}
\hline Enzymes & Subcellular localization & Enzymatic activities & $($ Refs.) \\
\hline ADPRT1a & - & Mono-ADP-ribosyltransferase & $(38)$ \\
ADPRT2 & - & Mono-ADP-ribosyltransferase & (38) \\
\hline
\end{tabular}

D, Sirtuins

\begin{tabular}{lccc}
\hline Enzymes & Subcellular localization & Enzymatic activities & $($ Refs.) \\
\hline SIRT1 & Nucleus & - & $(27,28)$ \\
SIRT2 & Nucleus/Cytoplasm & - & $(27,28)$ \\
SIRT3 & Mitochondria & Mono-ADP-ribosyltransferase & $(27,28)$ \\
SIRT4 & Mitochondria & - & $(25,32)$ \\
SIRT5 & Mitochondria & Mono-ADP-ribosyltransferase \\
SIRT6 & Nucleus & Mono-ADP-ribosyltransferase & $(26,33)$ \\
SIRT7 & Nucleus (nucleoli) & $(25)$
\end{tabular}

ARTDs, bacterial diphtheria toxin-like ADP-ribosyltransferases; ARTCs, clostridial C2 and C3 toxin-like ARTs.

physiological conditions are considered to function in supporting the conversion of the chromatin loop into its transcriptional active structure (70). ARTD14, as a mono-ADP-ribosyltransferase of histones, was indicated to interact with aryl hydrocarbon receptor (AHR) leading to decreased AHR transcriptional activity (28).

\section{Mono-ADP-ribosylation of histones in cell proliferation and differentiation}

Mono-ADP-ribosylation of histones may promote or inhibit cell proliferation. It has been indicated that the 
Table II. Mono-ADP- ribosylation of histones.

A, H1 substrate

\begin{tabular}{lclr}
\hline Modified amino acid & Enzymes & \multicolumn{1}{c}{ Effect of the reaction } \\
\hline E2, E14E116 and K213 & - & Regulation of H1-H1 interactions \\
R34 & - & Blocks the cAMP-dependent phosphorylation of histone H1 \\
Q/N & ARTD3 & DNA repair & $(36)$ \\
\hline
\end{tabular}

$\mathrm{B}, \mathrm{H} 2 \mathrm{~A}$ substrate

\begin{tabular}{lcl}
\hline Modified amino acid & Enzymes & \multicolumn{1}{c}{ Effect of the reaction } \\
\hline Unknown & Sir2 & Response to oxidative stress/DNA damage \\
Unknown & Sir2 & $\begin{array}{l}\text { Inhibition of histone acetylation/silencing } \\
\text { chromatin domains } \\
\text { R/E }\end{array}$ \\
\end{tabular}

C, H2B substrate

\begin{tabular}{|c|c|c|c|}
\hline Modified amino acid & Enzymes & Effect of the reaction & (Refs.) \\
\hline $\mathrm{E} 2$ & ARTD3/ARTD10 & Unknown & $(31,38,42)$ \\
\hline E18/E19 & ARTs, Adprt1a/Adprt2 & Response to oxidative stress/DNA damage & $(38,39)$ \\
\hline Unknown & Sir2 & $\begin{array}{l}\text { Inhibition of histone acetylation/silencing of } \\
\text { chromatin domains }\end{array}$ & $(43,68)$ \\
\hline
\end{tabular}

D, H3 substrate

\begin{tabular}{|c|c|c|c|}
\hline Modified amino acid & Enzymes & Effect of the reaction & (Refs.) \\
\hline Unknown & SIRT6, Sir2 & $\begin{array}{l}\text { Inhibition histone acetylation/silencing } \\
\text { chromatin domains }\end{array}$ & $(33,43)$ \\
\hline $\mathrm{R}$ & - & Cell proliferation & $(11,40)$ \\
\hline
\end{tabular}

E, H4 substrate

\begin{tabular}{|c|c|c|c|}
\hline Modified amino acid & Enzymes & Effect of the reaction & (Refs.) \\
\hline $\mathrm{R}$ & Sir2 & $\begin{array}{l}\text { Post-synthetic modification with acetylation of } \\
\text { core histones }\end{array}$ & $(27,28,40)$ \\
\hline Unknown & Sir2 & Response to oxidative stress/DNA damage & $(43)$ \\
\hline Unknown & ARTD10 & Unknown & $(42,66)$ \\
\hline Unknown & Sir2-relatedprotein & Inhibition histone acetylation & (43) \\
\hline
\end{tabular}

mono-ADP-ribosylation of histone 3 at R117 may accelerate the proliferation of colon carcinoma cells by regulating P300 to increase the expression level of cyclin D1 and c-myc (11). By contrast, other studies have indicated that after P815 mouse mastocytoma and K562 human chronic myelogenous leukemia cell lines were treated with $5 \mathrm{mM}$ butyrate or with serum-free media for blocking cell proliferation, the level of mono-ADP-ribosylated histones was higher compared with that in rapidly dividing cells. Of note, there were also no poly-ADP-ribosylated histones in the treated cells, while an increase in poly-ADP-ribosylated histones was observed in the rapidly dividing cells (69). The cycle of the conversion of poly-ADP-ribosylated histones to mono-ADP-ribosylated histones may be an important regulatory factor in cell proliferation. In addition, a study by our group indicated that arginine 117 of histone $\mathrm{H} 3$ in LoVo colon carcinoma cells with low differentiation were modified by mono-ADP-ribosylation, while SW480 cells with high differentiation were not (71), which suggested that mono-ADP-ribosylated histones may vary across different colorectal cancer cell lines with different degrees of malignancy, 
confirming the hypothesis that histone mono-ADP-ribosylation may have an important role in the development of tumors.

\section{Association between mono-ADP-ribosylation of histones and other histone modifications}

A wide range of histone modifications has been identified, which regulate signaling pathways in cell physiology and pathology. These modifications do not exist independently and there are connections among them. $\mathrm{H} 4$ is more likely to be mono-ADP-ribosylated while it is hyper-acetylated $(40,72)$. Mono-ADP-ribosylation of H4 by ARTD10 may occur when K5, K8 and/or K16 are modified by acetylation; however, these interrelations were indicated to be relatively weak. In addition, mono-ADP-ribosylation of H3 R117 affected the transcription and expression level of demethylase ten-eleven translocation 1 , thus regulating the methylation of tissue factor pathway inhibitor 2 in colorectal cancer (73). Furthermore, the decrease of mono-ADP-ribosylation of histones in colorectal carcinoma cells resulted in an increase of histone $\mathrm{H} 3$ trimethylated at lysine 4 and phosphatase and tensin homolog, thus reducing the phosphorylation of the PI3K/Akt signaling pathway. Mono-ADP-ribosylation of JHDM1A/KDM2A by SIRT6 led to an increase of histone $\mathrm{H} 3$ lysine 36 dimethylation levels to promote DNA repair (32). Methylation or acetylation of K20 in H4 may inhibit mono-ADP-ribosylation (29). When Arg34 of histone H1 is modified by mono-ADP-ribosylation, cyclic (c)AMP-dependent phosphorylation of histone $\mathrm{H} 1$ on Ser 38 may be inhibited (35). Arginine, which is located in the $\mathrm{NH}_{2}$-terminal of the phosphate-accepting serine residue, was indicated to be important for phosphorylation by cAMP-dependent protein kinase. Hence, the change in the function of the arginine residue by mono-ADP-ribosylation may affect the phosphorylation of histones. In yeast, histone acetylation may be inhibited by mono-ADP-ribosylation of histones, which is catalyzed by SIR 2 and may be responsible for inhibiting growth or silencing genes (74). It was also reported that reducing the ability of the mono-ADP-ribosyltranferase of SIR2 with a G270A mutation may not control gene silencing and it was hypothesized that histone acetylation may serve a bigger role rather than just in histone mono-ADP-ribosylation (75). Therefore, the efficiency of mono-ADP-ribosylation of histones by SIR2 requires further investigation.

\section{Hydrolytic enzymes of histone mono-ADP-ribosylation}

Mono-ADP-ribosylation is a reversible reaction, which may be hydrolyzed by Arg-specific mono-ADP-hydrolase, macroD1, macroD2, C6ORF130/TARG1 (76-78) and by serine mono ADP-ribosylhydrolase-3 (79). The content of macrodomain proteins primarily originates from viruses, such as $\alpha$-virus, hepatitis $E$ virus, severe acute respiratory syndrome coronavirus (SARS-CoV), feline infectious peritonitis virus and hCoV-229E macrodomains (80-82).

In a recent study, SARS-CoV-2 was reported to be able to remove mono-ADP-ribose (MAR) from a protein substrate (83). Whether the functions of histone modification were regulated by these hydrolytic enzymes requires further investigation. As the specific amino acid residues hydrolyzed by these hydrolases are different, the acceptor sites of histone
mono-ADP-ribosylation may be confirmed by the type of hydrolases able to hydrolyze the mono-ADP-ribosylation.

\section{Conclusion}

Mono-ADP-ribosylation has become a focus in the fields of immunity, inflammation, stress response, DNA damage response and cancer $(5,84,85)$. Different target proteins and even different amino acid residues may determine the functions of mono-ADP-ribosylation. However, the number of identified target proteins of mono-ADP-ribosylation remains low at present, owing to the limited and simplistic methods, not to mention the exact number and location of the acceptor sites.

Histones are major target proteins; however, knowledge regarding their function in pathophysiological processes is currently limited. Histone modifications, similar to acetylation, methylation and phosphorylation, have already been reported to participate in multiple processes, particularly in tumorigenesis. Studies have indicated that mono-ADP-ribosylation of histones is able to regulate the DNA damage response, transcription and cell proliferation, which are also important factors in tumorigenesis. The connections between histone mono-ADP-ribosylation and other well-known histone modifications indicate that the combined effects of these modifications may regulate pathophysiological processes.

\section{Acknowledgements}

Not applicable.

\section{Funding}

This study was supported by National High-Tech Research and Development Projects (863; grant no. 2012AA02A201), the Scientific Research Foundation of Chongqing Medical University (grant no. 201413), the Science and Technology Research Foundation of Chongqing Municipal Education Commission (grant no. KJQN201800435) and the National Nature Science Foundation of China (grant no. 30870946).

\section{Availability of data and materials}

The datasets used/or analyzed during the current study are available from the corresponding author on reasonable request.

\section{Authors' contributions}

JJZ and YT performed the literature search for relevant publications on the topic. YLW participated in drafting the manuscript and provided critical insight. JJZ and YT confirm the authenticity of all the raw data. All authors read and approved the final version of the manuscript.

\section{Ethics approval and consent to participate}

Not applicable.

\section{Patient consent for publication}

Not applicable. 


\section{Competing interests}

The authors declare that they have no competing interests.

\section{References}

1. Verma M, Maruvada $P$ and Srivastava S: Epigenetics and cancer Genes Dev 18: 2315-2335, 2016.

2. Shanmugam MK, Arfuso F, Arumugam S, Chinnathambi A Jinsong B, Warrier S, Wang LZ, Kumar AP, Ahn KS, Sethi G and Lakshmanan M: Role of novel histone modifications in cancer. Oncotarget 9: 11414-11426, 2017.

3. Wang R, Xin M, Li Y, Zhang P and Zhang M: The functions of histone modification enzymes in cancer. Curr Protein Pept Sci 17: 438-45, 2016.

4. FüllgrabeJ,Kavanagh Eand Joseph B: Histone onco-modifications. Oncogene 30: 3391-403, 2011.

5. Feijs KL, Verheugd $P$ and Lüscher B: Expanding functions of intracellular resident mono-ADP-ribosylation in cell physiology. FEBS J 280: 3519-3529, 2014.

6. Verheugd P, Forst AH, Milke L, Herzog N, Feijs KL, Kremmer E, Kleine $\mathrm{H}$ and Lüscher B: Regulation of NF-kappaB signalling by the mono-ADP-ribosyltransferase ARTD10. Nat Commun 4 : 1683,2013

7. Jwa M and Chang PE: PARP16 is a tail-anchored endoplasmic reticulum protein required for the PERK- and IRE1 $\alpha$-mediated unfolded protein response. Nat Cell Biol 14: 1223-1230, 2012.

8. Kistemaker HAV, Nardozza AP, Overkleeft HS van der Marel GA, Ladurner AG and Filippov DV: Synthesis and macrodomain binding of Mono-ADP-Ribosylated peptides. Angew Chem Int Ed Engl 55: 10634-10638, 2016.

9. Hottiger MO: ADP-ribosylation of histones by ARTD1: An additional module of the histone code? FEBS Lett 585: $1595-1599,2011$

10. Posavec Marjanović M, Crawford K and Ahel I: PARP, transcription and chromatin modeling. Semin Cell Dev Biol 63: 102-113, 2017.

11. Ling F, Tang Y, Li M, Li QS, Li X, Yang L, Zhao W, Jin CC, Zeng Z, Liu C, et al: Mono-ADP-ribosylation of histone 3 at arginine-117 promotes proliferation through its interaction with P300. Oncotarget 8: 72773-72787, 2017.

12. Verdone L, La Fortezza M, Ciccarone F, Caiafa P, Zampieri M and Caserta M: Poly(ADP-Ribosyl)ation affects histone acetylation and transcription. PLoS One 10: e0144287, 2015.

13. Kassner I, Andersson A, Fey M, Tomas M, Ferrando-May E and Hottiger MO: SET7/9-dependent methylation of ARTD1 at K508 stimulates poly-ADP-ribose formation after oxidative stress. Open Biol 3: 120173, 2013.

14. Tikoo K, Lau SS and Monks TJ: Histone H3 phosphorylation is coupled to poly-(ADP-ribosylation) during reactive oxygen species-induced cell death in renal proximal tubular epithelial cells. Mol Pharmacol 60: 394-402, 2001.

15. Mareike B, Laura E, Patricia V and Bernhard L: Intracellular Mono-ADP-ribosylation in signaling and disease. Cells 4 : 569-595, 2015

16. Girolamo MD and Fabrizio G: The ADP-Ribosyl-transferases diphtheria toxin-like (ARTDs) family: An overview. Challenges 9: 24, 2018.

17. Sadakierska-Chudy A and Filip MG: A comprehensive view of the epigenetic landscape. Part II: Histone post-translational modification, nucleosome level, and chromatin regulation by ncRNAs. Neurotox Res 27: 172-197, 2015.

18. Carter-O'Connell I and Cohen MS: Identifying direct protein targets of poly-ADP-ribose polymerases (PARPs) using engineered PARP variants-orthogonal nicotinamide adenine dinucleotide (NAD+) analog pairs. Curr Protoc Chem Biol 7: $121-139,2015$

19. Wang S, Xue X, Pharmacy SO and University CP: PARP family and clinically used PARP Inhibitors. Guangdong Chemical Industry 46: 134-136, 2019 (In Chinese).

20. Pinto AF and Schüler H: Comparative structural analysis of the putative mono-ADP-ribosyltransferases of the ARTD/PARP family. Curr Top Microbiol Immunol 384: 153-166, 2015.

21. Loseva O, Jemth AS, Bryant HE, Schüler H, Lehtiö L, Karlberg T and Helleday T: PARP-3 Is a Mono-ADP-ribosylase That Activates PARP-1 in the Absence of DNA. J Biol Chem 285: 8054-8060, 2010.

22. Krska D, Ravulapalli R, Fieldhouse RJ,Lugo MR and Merrill AR: C3larvin Toxin, an ADP-ribosyltransferase from Paenibacillus larvae. J Biol Chem 290: 1639-1653, 2015.
23. Prisilla A and Chellapandi P: Structure, function and evolution of clostridium botulinum $\mathrm{C} 2$ and $\mathrm{C} 3$ toxins: Insight to poultry and veterinary vaccines. Curr Protn Pept 18: 412-424, 2017.

24. Li S and Zheng W: Mammalian sirtuins SIRT4 and SIRT7. Prog Mol Biol Transl Sci 154: 147-168, 2018.

25. Rahnasto-Rilla M, Lahtela-Kakkonen M and Moaddel R Sirtuin 6 (SIRT6) activity assays. Methods Mol Biol 1436 259-269, 2016.

26. Xinxin QI and Li S: Sirtuin family and its biological characteristics. Acta Med Sin, 2016.

27. Balaiya S, Abu-Amero KK, Kondkar AA and Chalam KV: Sirtuins expression and their role in retinal diseases. Oxid Med Cell Longev 2017: 3187594, 2017.

28. MacPherson L, Tamblyn L, Rajendra S, Bralha F, McPherson JP and Matthews $\mathrm{J}$ : 2,3,7,8-Tetrachlorodibenzo-p-dioxin poly(ADP-ribose) polymerase (TiPARP, ARTD14) is a mono-ADP-ribosyltransferase and repressor of aryl hydrocarbon receptor transactivation. Nuclc Acids Res 41: 1604-1621, 2013.

29. Feijs K: Characterization of the mono-ADP-ribosylation by ARTD10: Substrates, consequences and reversibility. Hochschulbibliothek der Rheinisch-Westfälischen Technischen Hochschule Aachen, 2012.

30. Grundy GJ, Polo LM, Zeng Z, Rulten S, Hoch NC, Paomephan P, $\mathrm{Xu} \mathrm{YQ}$, Sweet SM, Thorne AW, Oliver AW, et al: PARP3 is a sensor of nicked nucleosomes and monoribosylates histone H2B(Glu2). Nat Commun 7: 12404, 2016.

31. Ahuja N, Schwer B, Carobbio S, Waltregny D, North BJ, Castronovo V, Maechler P and Verdin E: Regulation of insulin secretion by SIRT4, a mitochondrial ADP-ribosyltransferase. J Biol Chem 282: 33583-33592, 2007.

32. Rezazadeh S, Yang D, Biashad SA, Firsanov D and Gorbunova V: SIRT6 mono-ADP ribosylates KDM2A to locally increase H3K36me2 at DNA damage sites to inhibit transcription and promote repair. Aging 12: 11165-11184, 2020.

33. Hassa PO, Haenni SS, Elser M and Hottiger MO: Nuclear ADP-ribosylation reactions in mammalian cells: Where are we today and where are we going? Microbiol Mol Biol Rev 70: 789-829, 2006.

34. Ogata N, Ueda K, Kagamiyama $\mathrm{H}$ and Hayaishi $\mathrm{O}$ : ADP-ribosylation of histone $\mathrm{H} 1$. Identification of glutamic acid residues 2, 14, and the $\mathrm{COOH}$-terminal lysine residue as modification sites. J Biol Chem 255: 7616-7620, 1980.

35. Ushiroyama T, Tanigawa Y, Tsuchiya M, Matsuura R, Ueki M, Sugimoto $O$ and Shimoyama $M$ : Amino acid sequence of histone $\mathrm{H} 1$ at the ADP-ribose-accepting site and ADP-ribose $\mathrm{X}$ histone-H1 adduct as an inhibitor of cyclic-AMP-dependent phosphorylation. Eur J Biochem 151: 173-177, 2010.

36. Riquelme PT, Burzio LO and Koide SS: ADP ribosylation of rat liver lysine-rich histone in vitro. J Biol Chem 254: 3018-3028, 1979.

37. Ogata N, Ueda K and Hayaishi O: ADP-ribosylation of histone $\mathrm{H} 2 \mathrm{~B}$. Identification of glutamic acid residue 2 as the modification site. J Biol Chem 255: 7610-7615, 1980.

38. Rakhimova A, Ura S, Hsu DW, Wang HY, Pears CJ and Lakin ND: Site-specific ADP-ribosylation of histone H2B in response to DNA double strand breaks. Sci Rep 7: 43750, 2017.

39. Golderer G and Gröbner P: ADP-ribosylation of core histones and their acetylated subspecies. Biochem J 277: 607-610, 1991.

40. Dan MB: European geosciences union general assembly. Nuclc Acids Res 38: 6350-6362, 2016.

41. Kleine H, Poreba E, Lesniewicz K, Hassa PO, Hottiger MO, Litchfield DW, Shilton B and Lüscher B: Substrate-assisted catalysis by PARP10 limits its activity to mono-ADP-ribosylation. Mol Cell 32: 57-69, 2008.

42. García-Salcedo JA, Gijón P, Nolan DP, Tebabi P and Pays E: A chromosomal SIR2 homologue with both histone NAD-dependent ADP-ribosyltransferase and deacetylase activities is involved in DNA repair in Trypanosoma brucei. EMBO J 22: 5851-5862, 2003

43. Graves DJ, Huiatt TW, Zhou H, Huang HY and Mcmahon KK: Regulatory role of arginine-specific mono(ADP-Ribosyl)transferase in muscle cells. Adv Exp Med Biol 419: 305-313, 1997.

44. Schwab CJ, Colville MJ, Fullerton AT and Mcmahon KK: Evidence of endogenous mono-ADP-ribosylation of cardiac proteins via anti-ADP-ribosylarginine immunoreactivity. Proc Soc Exp Biol Med 223: 389-396, 2010.

45. Meyer T and Hilz H: Production of anti-(ADP-ribose) antibodies with the aid of a dinucleotide-pyrophosphatase-resistant hapten and their application for the detection of mono(ADP-ribosyl)ated polypeptides. Eur J Biochem 155: 157-165, 2010. 
46. Osago H, Terashima M, Hara N, Yamada K and Tsuchiya M: A new detection method for arginine-specific ADP-ribosylation of protein-a combinational use of anti-ADP-ribosylarginine antibody and ADP-ribosylarginine hydrolase. J Biochem Biophys Methods 70: 1014-1019, 2008.

47. van der Heden van Noort GJ: Chemical tools to study protein ADP-ribosylation. ACS Omega 5: 1743-1751, 2020.

48. Liu Q, Marel GAVD and Filippov DV: Chemical ADP-ribosylation: Mono-ADPr-peptides and oligo-ADP-ribose. Organ Biomol Chem 17: 5460-5474, 2019.

49. Moyle PM and Muir TW: Method for the synthesis of mono-ADP-ribose conjugated peptides. J Am Chem Soc 132: 15878-15880, 2010.

50. Vivelo CA and Leung AK: Proteomics approaches to identify mono-(ADP-ribosyl)ated and poly(ADP-ribosyl)ated proteins. Proteomics 15: 203-217, 2015.

51. Lu AZ, Abo R, Ren Y, Gui B, Mo JR, Blackwell D, Wigle T, Keilhack H and Niepel M: Enabling drug discovery for the PARP protein family through the detection of mono-ADP-ribosylation. Biochem Pharmacol 168: 97-106, 2019.

52. Han W, Li X and Fu X: The macro domain protein family: Structure, functions, and their potential therapeutic implications. Mutat Res 727: 86-103, 2011.

53. Feijs KLH, Forst AH, Verheugd $P$ and Lüscher $B$ Macrodomain-containing proteins: Regulating new intracellular functions of mono(ADP-ribosyl)ation. Nat Rev Mol Cell Biol 14 443-451, 2013.

54. Forst AH, Karlberg T, Herzog N, Thorsell AG, Gross A, Feijs KL, Verheugd P, Kursula P, Nijmeijer B, Kremmer E, et al: Recognition of mono-ADP-ribosylated ARTD10 substrates by ARTD8 macrodomains Structure 21: 426-475, 2013.

55. Osago H, Yamada K, Shibata T, Yoshino KI, Hara N and Tsuchiya M: Precursor ion scanning and sequencing of arginine-ADP-ribosylated peptide by mass spectrometry. Anal Biochem 393: 248-254, 2009.

56. Perkins DN, Pappin DJC, Creasy DM and Cottrell JS Probability-based protein identification by searching sequence databases using mass spectrometry data. Electrophoresis 20 3551-3567. Electrophoresis 20: 3551-3567, 1999.

57. Chen J, Lam AT and Zhang Y: A macrodomain-linked immunosorbent assay (MLISA) for mono-ADP-ribosyltransferases. Anal Biochem 543: 132-139, 2017.

58. Leutert M, Bilan V, Gehrig P and Hottiger MO: Identification of ADP-ribose acceptor sites on in vitro modified proteins by liquid chromatograph-tandem mass spectrometry. Methods Mol Biol 1608: 137-148, 2017.

59. Larsen SC, Leutert M, Bilan V, Martello R, Jungmichel S, Young C, Hottiger MO and Nielsen ML: Proteome-wide identification of in vivo ADP-ribose acceptor sites by liquid chromatography-tandem mass spectrometry. Methods Mol Biol 1608: 149-162, 2017.

60. Daniels CM, Ong SE and Leung AK: Phosphoproteomic approach to characterize protein mono- and poly(ADP-ribosyl) ation sites from cells. J Proteome Res 13: 3510-3522, 2014.

61. Morgan RK and Cohen MS: A clickable aminooxy probe for monitoring cellular ADP-ribosylation. ACS Chem Biol 10: $1778-1784,2015$

62. Messner S and Hottiger MO: Histone ADP-ribosylation in DNA repair, replication and transcription. Trends Cell Biol 21: 534-542, 2011

63. Karch KR, Langelier MF, Pascal JM and Garcia BA: The nucleosomal surface is the main target of histone ADP-ribosylation in response to DNA damage. Mol Biosys 13: 2660-2671, 2017.

64. Adamietz $P$ and Rudolph A: ADP-ribosylation of nuclear proteins in vivo. Identification of histone $\mathrm{H} 2 \mathrm{~B}$ as a major acceptor for mono- and poly(ADP-ribose) in dimethyl sulfate-treated hepatoma AH 7974 cells. J Biol Chem 259: 6841-6846, 1984.

65. Kreimeyer A, Adamietz $\mathrm{P}$ and Hilz H: Alkylation-induced mono(ADP-ribosyl)-histones H1 and H2B Hydroxylamine-resistant linkage in hepatoma cells. Biol Chem 366: 537-544, 1985.

66. Kreimeyer A, Wielckens K, Adamietz P and Hilz H: DNA repair-associated ADP-ribosylation in vivo. Modification of histone $\mathrm{H} 1$ differs from that of the principal acceptor proteins. J Biol Chem 259: 890-896, 1984
67. Rulten SL, Fisher AEO, Robert I, Zuma MC, Rouleau M, Ju LM Poirier G, Reina-San-Martin B and Caldecott KW: PARP-3 and APLF function together to accelerate nonhomologous end-joining. Mol Cell 41: 33-45, 2011.

68. Boulikas T: DNA strand breaks alter histone ADP-ribosylation. Proc Natl Acad Sci USA 86: 3499-3503, 1989.

69. Boulikas T: Poly(ADP-ribosylated) histones in chromatin replication. J Biol Chem 265: 14638-14647, 1990.

70. Boulikas T: Relation between carcinogenesis, chromatin structure and poly(ADP-ribosylation) (review). Anticancer Res 11: 489-527, 1991.

71. Zhang NN, Lin T, Xiao M, Li QS, Li X, Yang L, Wang CL and Wang YL: Transcriptome sequencing analysis of monoADPribosylation in colorectal cancer cells. Oncol Rep 43: 1413-1428, 2020.

72. Böhm L, Schneeweiss FA, Sharan RN and Feinendegen LE: Influence of histone acetylation on the modification of cytoplasmic and nuclear proteins by ADP-ribosylation in response to free radicals. Biochim Biophys Acta 1334: 149-154, 1997.

73. Li M, Tang Y, Li Q, Xiao M, Yang Y and Wang Y: Mono-ADP-ribosylation of H3R117 traps 5mC hydroxylase TET1 to impair demethylation of tumor suppressor gene TFPI2. Oncogene 38: 3488-3503, 2019.

74. Tanny JC, Dowd GJ, Huang J, Hilz H and Moazed D: An enzymatic activity in the yeast Sir2 protein that is essential for gene silencing. Cell 99: 735-45, 1999.

75. Michan S and Sinclair D: Sirtuins in mammals: Insights into their biological function. Biochem J 404: 1-13, 2007.

76. Laing S, Unger M, Koch-Nolte F and Haag F: ADP-ribosylation of arginine. Amino Acids 41: 257-69, 2011.

77. Stevens LA, Kato J, Kasamatsu A, Oda H, Lee DY and Moss J: The ARH and macrodomain families of $\alpha$-ADP-ribose-acceptor hydrolases catalyze $\alpha-\mathrm{NAD}^{+}$hydrolysis. ACS Chem Biol 14: 2576-2584, 2019

78. Thomas A, Deeksha M, Kerryanne C, Luca P, Andreja M and Ivan A: MacroD1 is a promiscuous ADP-Ribosyl hydrolase localized to mitochondria. Front Microbiol 9: 20, 2018.

79. Abplanalp J, Leutert M, Frugier E, Nowak K, Feurer R, Kato J, Kistemaker HVA, Filippov DV, Moss J, Caflisch A and Hottiger MO: Proteomic analyses identify ARH3 as a serine mono-ADP-ribosylhydrolase. Nat Commun 8: 2055, 2017.

80. Fehr AR, Channappanavar R, Jankevicius G, Fett C, Zhao J, Athmer J, Meyerholz DK, Ahel I and Perlman S: The conserved coronavirus macrodomain promotes virulence and suppresses the innate immune response during severe acute respiratory syndrome coronavirus infection. mBio 7: e01721-16, 2016.

81. Eckei L, Krieg S, Bütepage M, Lehmann A, Gross A, Lippok BE, Grimm AR, Kümmerer BM, Rossetti G, Lüscher B and Verheugd P: The conserved macrodomains of the non-structural proteins of Chikungunya virus and other pathogenic positive strand RNA viruses function as mono-ADP-ribosylhydrolases. Sci Rep 7: 41746, 2017.

82. Li C, Debing Y, Jankevicius G, Neyts J, Ahel I, Coutard B and Canard B: Viral macro domains reverse protein ADP-ribosylation. J Virol 90: 8478-8486, 2016.

83. Alhammad YMO, Kashipathy MM, Roy A, Gagné JP, McDonald P, Gao P, Nonfoux L, Battaile KP, Johnson DK, Holmstrom ED, et al: The SARS-CoV-2 conserved macrodomain is a highly efficient ADP-ribosylhydrolase enzyme. bioRxiv: 2020.05.11.089375, 2020

84. Munnur D and Ahel I: Reversible mono-ADP-ribosylation of DNA breaks. FEBS J 284: 4002-4016, 2017.

85. Scarpa ES, Fabrizio G and Di Girolamo M: A role of intracellular mono-ADP-ribosylation in cancer biology. FEBS J 280 3551-3562, 2013

This work is licensed under a Creative Commons Attribution-NonCommercial-NoDerivatives 4.0 International (CC BY-NC-ND 4.0) License. 